\title{
Possible acetaminophen poisoning complicated by puerperal sepsis
}

Arop M. D. Kual

Anaesthesiologist, Francistown Academic

Hospital, Francistown, Botswana

Correspondence:

Arop M. D. Kual

safeanaesthesiaservices@yahoo.com

Submitted: June 2021

Accepted: August 2021

Published: November 2021
Citation: Kual, Possible acetaminophen poisoning complicated by puerperal sepsis. South Sudan Medical Journal 2021;14(4):138-141 (C) 2021 The Author (s) License: This is an open access article under CC BY-NC DOI: https://dx.doi. org/10.4314/ssmj.v14i4.8

\section{Abstract}

Acetaminophen (paracetamol) toxicity is an important cause of morbidity and mortality worldwide. It is estimated that poisoning contributes to more than 1 million poisonings and up to half a million deaths each year, yet it is preventable and treatable.

Acetaminophen toxicity is the second most common cause of liver transplantation worldwide.

However, studies concerning poisoning in Botswana are scant, although it has been reported that poisoning contributes $6.7 \%$ of the total injuries and is ranked third next to falls and automobile accidents among external causes of mortality.

This case report reviews the aetiology, evaluation, and treatment of acetaminophen overdose.

Key words: Acetaminophen / Paracetamol toxicity, acetaminophen overdose, acute liver failure, $\mathrm{N}$-acetylcysteine, liver hepatotoxicity, hepatic encephalopathy, liver transplant.

\section{Introduction}

Acetaminophen (paracetamol) toxicity is a common medical emergency with serious morbidity and mortality worldwide. ${ }^{[1]}$

In Botswana, the highest number of cases of poisoning are caused by acetaminophen if taken in excess of $10-15 \mathrm{gms}^{[2]}$

Single or repeated doses totalling $10-15 \mathrm{~g}$ or $150 \mathrm{mg} / \mathrm{kg}$ of acetaminophen taken within 24 hours may cause severe hepatotoxicity and, less frequently, acute kidney injury (AKI).

The objectives of this paper are to

- Review the pathophysiology of acetaminophen toxicity.

- Describe the four clinical stages of acetaminophen toxicity.

- Identify the treatment strategies for an alert patient who presents within an hour of overdose versus a patient who presents after two hours.

\section{Case Report}

We describe a 25-year-old female referred to our hospital's intensive care unit (ICU) with suspected acetaminophen toxicity.

The patient had acute liver failure (ALF), acute kidney injury (AKI) with oliguria, incomplete abortion, underlying hydrocephalus managed with ventriculoperitoneal shunt (VP shunt), blindness and depression.

The patient swallowed an unknown number of acetaminophen tablets a day before presentation as reported by the mother who additionally said the patient had episodes of headache. Acetaminophen, as an over counter medication, had 
Table 1. Glasgow Coma Scale (GCS)

Eye Opening Response
- $\quad$ Spontaneous--open with blinking at baseline 4
- $\quad$ To verbal stimuli, command, speech 3 points
- $\quad$ No response 1 point
Verbal Response
- $\quad$ Oriented 5 points
- $\quad$ Confused conversation, but able to answer
- $\quad$ Inapstions 4 points
- $\quad$ Incomprehensible speech 2 points
- $\quad$ No response 1 point
Motor Response
- $\quad$ Obeys commands for movement 6 points
- $\quad$ Purposeful movement to painful stimulus 5 points
- $\quad$ Withdraws in response to pain 4 points
- $\quad$ Flexion in response to pain (decorticate posturing)
- $\quad$ Extension response in response to pain
- $\quad$ No response 1 point

been bought by a relative. The mother did not know how many tablets had been obtained and ingested. The tablet bottle was not brought to the hospital. The patient had been initially managed at the referring hospital for two days before transfer to our ICU.

The patient had severe hepatic dysfunction with elevated liver enzymes and altered sensorium consistent with hepatic encephalopathy. Serum aspartate aminotransferase (AST/SGOT) was initially $300 \mathrm{U} / \mathrm{L}$ (normal range: 10 - 34) while serum alanine transferase (ALT/SGPT) was not measured in the referring hospital. The patient had right upper quadrant tenderness on deep palpation with flapping tremors but there was no jaundice. She rapidly developed tachypnoea and respiratory distress with foaming from the mouth.

Upon arrival to our ICU, the patient's Glasgow Coma Scale (GCS) fell to $6 / 15$, respiratory rate of $54 \mathrm{~b} / \mathrm{m}$, heart rate of $140 \mathrm{bpm}$, blood pressure fluctuating between 110 $130 / 40-60 \mathrm{mmHg}$ and saturation of $90 \%$ on high flow oxygen. The GCS is the most common scoring system used to describe the level of consciousness in a person -Table 1. A urinary catheter drained $100 \mathrm{ml}$ of concentrated urine.

A person's GCS score (Table 1) can range from 3 (completely unresponsive) to 15 (responsive). This score is used to guide immediate medical care.

In view of the respiratory distress and falling GCS, the patient was intubated and mechanically ventilated.
The blood pressure dropped to $80 / 40 \mathrm{mmHg}$ which did not respond to IV fluid therapy (2 litres of normal saline $0.9 \%)$. Adrenaline infusion was started followed by dopamine infusion, and N-acetylcysteine (NAC) administered after central venous cannulation of the right subclavian vein. The plasma level of acetaminophen could not be measured in our facility.

$\mathrm{N}$-acetylcysteine (NAC) is an amino acid produced from L-cysteine that is used as a dietary supplement. It is utilized as an acetaminophen overdose antidote. It is thought to reduce DNA damage as an antioxidant. NAC is also used to support healthy immunological function and has liver-protective effects.

Tables 2 and 3 show the values for the routine laboratory tests done.

The elevated serum creatinine suggested acute kidney injury and the high liver transaminases suggest severe liver damage. The patient also showed electrolytes imbalance of hyperchloraemia indicating the inability of her kidneys to regulate electrolytes due to dehydration and probably severe acute kidney injury. Prothrombin time done at the referring hospital revealed an elevated level of $26.3 \mathrm{sec}$. The INR (2.61) was raised above normal reference range. The patient was given clexane $40 \mathrm{mg}$ subcutaneously daily as prophylaxis against deep venous thrombosis (DVT).

She remained critically ill, oliguric, tachycardic, hypotensive on high dose of vasopressor.

Ultrasonography of the abdomen, revealed increased endometrial thickness and retained products of conception. A pelvic examination showed foul smelling discharge.

The patient presented with fever $39.4^{\circ} \mathrm{C}$ and hypotension $82 / 40 \mathrm{~mm} \mathrm{Hg}$, HR of $125 \mathrm{bpm}$, generalized malaise, and altered mentation suggesting sepsis. On arrival she was somnolent. She had a respiratory rate of 40 per minute, and a pulse oximetry of $92 \%$ on mechanical ventilation. Physical examination showed dry mucous membranes, tachycardia and regular rhythm, tachypnoea and normal pulmonary breath sounds. She was initially in pain as reported by the mother prior to her consumption of the unspecified number of acetaminophen tablets. We considered that acetaminophen poisoning might have been the leading primary insult, with sepsis complicating the picture.

Her urine and serum human chorionic gonadotrophin (HCG) were positive suggestive of a missed/incomplete abortion. Antibiotics (IV ceftriaxone $1 \mathrm{~g}$ daily and IV infusion of metronidazole $500 \mathrm{mg}$ three times daily) were prescribed with NAC. The gynaecologist was consulted who agreed with the likelihood of missed abortion.

The patient's condition deteriorated with falling blood 
pressure to $70 / 35 \mathrm{mmHg}$ and oxygen desaturation $<90 \%$. She was given one litre boluses of normal saline $0.9 \%$, adrenaline $6 \mathrm{mcg} / \mathrm{min}(90 \mathrm{ml} / \mathrm{hr})$ and dopamine infusions $50 \mathrm{mcg} / \mathrm{kg} / \mathrm{min}$ but unfortunately, she died on day 3 after admission to the ICU. The relatives refused consent for post-mortem examination.

\section{Discussion}

\section{Review of pathophysiology}

Within 24 hours following an acetaminophen overdose, anorexia, nausea, and vomiting appear. ${ }^{[3]}$ Hepatotoxicity may not show up in clinical tests for 4 to 6 days after ingestion. when acetaminophen levels are no longer detectable Necrosis around the central vein is a common lesion.

The liver enlarges and becomes tender. The liver transaminases (AST and ALT) rise for up to two weeks before returning to normal. Transaminase levels above 1,000 IU/L reflect severe liver damage. ${ }^{[4]}$

The bilirubin levels are typically only moderately elevated. Hepatic clotting factor synthesis is reduced, and prothrombin time is prolonged. It has been recorded that disseminated intravascular coagulation (DIC) occurs in the presence of hepatic necrosis. ${ }^{[5]}$

Acute kidney injury (AKI) caused by acetaminophen poisoning may occur alone or in combination with hepatic necrosis. Urine production varies between patients. Acute tubular necrosis (ATN) can be distinguished from hepatorenal syndrome (HRS) and prerenal azotaemia based on urinary findings.

Urinary granular casts with or without haematuria or pyuria are common in ATN. Urine osmolality is usually similar to plasma osmolality, but urine sodium concentration is usually greater than $20 \mathrm{mmol} / \mathrm{L}$. Urinary particles such as tubular epithelial cell casts and/or granular casts in the urine sediment are common, urinary sodium is low (less than $10 \mathrm{mmol} / \mathrm{L}$ ), and osmolality is far higher than plasma osmolality in HRS and prerenal azotaemia.

As seen in our case, renal dysfunction also occurs alongside hepatic encephalopathy in serious overdoses, and may necessitate dialysis.

\section{Stages of acetaminophen toxicity}

The clinical course of acetaminophen toxicity is divided into four stages.

- During the first stage (30 min to 24 hours), the patient may be asymptomatic or may have emesis.

- In the second stage (18 hours to 72 hours), there may be emesis plus right upper quadrant pain and hypotension.

- In the third stage (72 hours to 96 hours),
Table 2. Complete Blood Count

\begin{tabular}{lll} 
Test & Laboratory result & Reference range \\
\hline WBC & $9.42 \times 109 / \mathrm{L}$ & $4000-11000$ \\
$\mathrm{RBC}$ & $3.37 \times 1012 / \mathrm{L}$ & $3.5-5.4$ \\
$\mathrm{Hb}$ & $14.7 \mathrm{~g} / \mathrm{dL}$ & $11.5-16.5$ \\
$\mathrm{PCV} / \mathrm{HCT}$ & $46.3 \%$ & $33-48$ \\
$\mathrm{MCV}$ & $86.2 \mathrm{fL}$ & $83-99$ \\
$\mathrm{MCH}$ & $27.4 \mathrm{pg}$ & $27-31$ \\
Platelets & $149 \times 109 / \mathrm{L}$ & $150-400$ \\
Neutrophils & $7.27 \times 109 / \mathrm{L}$ & $2.0-7.5$ \\
Lymphocytes & $1.71 \times 109 / \mathrm{L}$ & $1.0-4.0$ \\
Monocytes & $0.43 \times 109 / \mathrm{L}$ & $0.1-0.8$ \\
Eosinophils & $0.00 \times 109 / \mathrm{L}$ & $0.0-0.4$ \\
Basophils & $0.01 \times 109 / \mathrm{L}$ & $0.0-0.2$
\end{tabular}

Table 3. Serum Urea, Electrolytes, Creatinine and Liver Transaminases

\begin{tabular}{lll} 
Test (Serum) & Laboratory result & Reference range \\
\hline Urea & $8.48 \mathrm{mmol}$ & $2.0-7.0$ \\
Creatinine & $283.3 \mathrm{umol} / \mathrm{L}$ & $44-106$ \\
Sodium & $150.3 \mathrm{mmol} / \mathrm{L}$ & $135-145$ \\
Potassium & $3.12 \mathrm{mmol} / \mathrm{L}$ & $3.5-5.1$ \\
Chloride & $116.4 \mathrm{mmol} / \mathrm{L}$ & $95-108$ \\
Total bilirubin & $164.2 \mathrm{umol} / \mathrm{L}$ & $0.1-21.0$ \\
Aspartate & $1285 \mathrm{U} / \mathrm{L}$ & $10-34$ \\
transferase (AST) & & \\
Alanine & $1100 \mathrm{U} / \mathrm{L}$ & $11-41$ \\
transferase (ALT) & & \\
Total protein & $50.8 \mathrm{~g} / \mathrm{L}$ & $60-80$ \\
Albumen & $21.7 \mathrm{~g} / \mathrm{L}$ & $15-55$ \\
Eosinophils & $0.00 \mathrm{X} 109 / \mathrm{L}$ & $0.0-0.4$ \\
Basophils & $0.01 \mathrm{X} 109 / \mathrm{L}$ & $0.0-0.2$
\end{tabular}

liver dysfunction is significant with renal failure, coagulopathies, metabolic acidosis, and encephalopathy. Gastrointestinal (GI) symptoms reappear, and death is most common at this stage.

- The fourth stage (4 days to 3 weeks) is marked by recovery.

\section{Treatment of acetaminophen toxicity}

An IV acetaminophen overdose is evaluated and treated similarly to an oral acetaminophen overdose. After ingestion, most emergency departments begin with gastrointestinal (GI) decontamination agents. If the patient is awake and presents within one hour of ingestion, oral activated charcoal is given. Acetaminophen is easily absorbed by activated charcoal. 


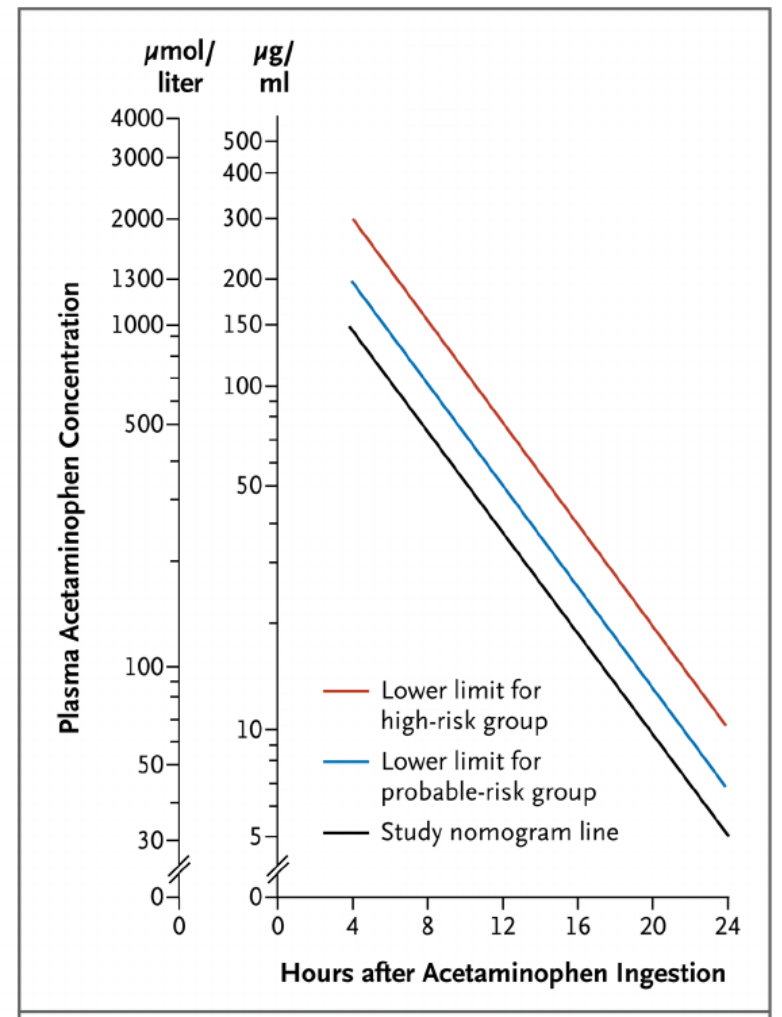

Figure 1. Rumack-Matthew Nomogram published with permission New England Journal of Medicine 359(3):285-92 DOI:10.1056/NEJMct0708278 July 2008 PubMed

If given within one hour of ingestion, or later if the ingestion contains an agent that delays gastric emptying or slows GI motility such as levodopa and nicotine, the activated charcoal may provide a major treatment advantage. After being medically approved, patients with acetaminophen concentrations below the "possible" line for hepatotoxicity on the Rumack-Matthew nomogram may be discharged.

The Rumack-Matthew nomogram (also known as the acetaminophen-toxicity nomogram, see Figure 1) plots serum acetaminophen concentration against time after ingestion to predict potential liver toxicity and to help the clinician decide whether or not to treat with NAC. It's a logarithmic graph that starts 4 hours after ingestion, after absorption is thought to be possible. ${ }^{[6]}$

This nomogram enables the treatment of acetaminophen overdose in a timely manner. A plasma and liver acetaminophen-protein (APAP) adduct levels of 140-150 $\mathrm{mcg} / \mathrm{mL}$ at 4 hours after ingestion usually indicates that NAC treatment is needed. When given within 8 hours of an acute acetaminophen ingestion, NAC is nearly 100 percent hepatoprotective. NAC is safe to take orally and intravenously.

The following is the FDA-approved NAC (Mucomyst) oral administration regimen: To make a $5 \%$ solution, dilute the $20 \%$ solution $1: 3$ with cola, orange juice, or another soft drink. For a total treatment of 72 hours, use within 1 hour of preparation.

NAC (Acetadote) IV: The IV version of NAC (Acetadote) is also used in many emergency rooms to treat acetaminophen ingestion. If the patient is unable to tolerate oral NAC due to emesis that is resistant to antiemetics, the IV formulation of NAC is favoured. ${ }^{[7]}$

For the loading dose, dilute $150 \mathrm{mg} / \mathrm{kg}$ (maximum $15 \mathrm{~g}$ ) in $200 \mathrm{~mL}$ of $5 \%$ dextrose in water (D5W) and infuse for 60 minutes. Dilute $50 \mathrm{mg} / \mathrm{kg}$ (maximum $5 \mathrm{~g}$ ) in D5W $500 \mathrm{~mL}$ for the second dose and infuse for 4 hours. Dilute $100 \mathrm{mg} / \mathrm{kg}$ (maximum $10 \mathrm{~g}$ ) in D5W $1,000 \mathrm{~mL}$ for the third dose and infuse for 16 hours to prevent fluid overload in patients weighing less than $40 \mathrm{~kg}$ and those who need dialysis.

Liver transplantation is the final resort in cases of serious hepatotoxicity that has progressed to liver failure.

\section{Conclusion}

Acetaminophen toxicity is a most common medical emergency that leads to morbidity and mortality around the world.

Following an acute overdose, severe toxicity may be mitigated or avoided with early diagnosis and prompt administration of NAC. Late presentation may lead to severe liver damage.

\section{References}

1. Van Hoving D, Hunter L, Gerber R, Lategan H, Marks C. The burden of intentional self-poisoning on a districthospital public hospital in Cape Town, South Africa. Afr J Emerg Med 2018;8(3):79-83.

2. Kasule M, Malangu N. Profile of acute poisoning in three health districts of Botswana. Afr J Prim Health Care Fam Med 2009;1:10.

3. Hodgman MJ, Garrard AR. A review of acetaminophen poisoning. Crit. Care Clin. 2012;28:499-516.

4. Rumack BH, Matthew H. Acetaminophen poisoning and toxicity. Pediatrics 1975;55:871-876.

5. Kramer J, Otten HM, Levi M, ten Cate H. The association of disseminated intravascular coagulation with specific diseases. Éditions Scientifiques et Médicales Elsevier SAS. 2002;576.

6. Dart RC, Erdman AR, Olson KR, Christianson G, Manoguerra AS, Chyka PA, et al. Acetaminophen poisoning: an evidence-based consensus guideline for outof-hospital management. Clin Toxicol (Phila) 2006;44:118. doi: 10.1080/15563650500394571.

7. Kanter MZ. Comparison of oral and i.v. acetylcysteine in the treatment of acetaminophen poisoning. Am J Health Syst Pharm. 2006;63:1821-1827 